\title{
SCHEDULING SCIENTIFIC WORKFLOW ON CLOUD USING META-HEURISTIC STATIC SCHEDULING ALGORITHM
}

\author{
AMBIKA AGGARWAL ${ }^{1}$, Dr. PRITI DIMRI ${ }^{2} \&$ Dr. AMIT AGARWAL ${ }^{3}$ \\ ${ }^{I}$ Assistant Professor, School of Computer Science, University of Petroleum and Energy Studies, \\ Dehradun, Uttarakhand, India \\ ${ }^{2}$ Associate Professor, Department of Computer Applications, GBPEC, Ghurdauri, Pauri, Uttarakhand, India \\ ${ }^{3}$ Professor and Director, Dr. APJ Abdul Kalam Institute of Technology, Tanakpur, Uttarakhand, India
}

\begin{abstract}
Workflow has reduced the complexity of scientific applications. Other than Grid and Cluster, Cloud Computing is an ideal platform for an efficient workflow deployment. But due to its dynamic variation in VMs, many challenges come across it. Quality of Service (QoS) in terms of cost and execution time is a key issue in workflow scheduling. In this paper, the authors have proposed a scheduler that is inspired by PSO (particle swarm optimization) and works on a given deadline neglecting its constraint. The technique used is the meta-heuristic approach and results have been simulated in Cloud Sim and experimental results show better performance of the proposed method as compared to other state -of-artalgorithms.
\end{abstract}

KEYWORDS: Scientific Workflow, Cloud Computing, Virtual Machines, Resource Provisioning, Deadline Constraint

Received: May 13, 2020; Accepted: Jun 03, 2020; Published: Aug 03, 2020; Paper Id.: IJMPERDJUN2020574

\section{INTRODUCTION}

Scientific workflow can be understood as a set of large-scale scientific experiments, which are executed using distributed platforms. Workflows play a vital role in setting up the objectives for scientists of various areas. Thus, workflow gives a programming platform to develop applications with platform independence [1].

Services like SGI Cap [2] provide a programming interface that can be used by the scientific community for creating scientific gateways.

Technologies like Cloud Computing provide ease for the smooth functioning of these scientific applications. A cloud environment consists of three architectural layers named as Software as a Service(SaaS), Plat form as a Service (PaaS), and Infrastructure as a Service(IaaS). In specific terms, IaaS layer provides the low-cost workflow deployment infrastructure and also provides access to the cluster of Virtual Machines (VM) that can be used on pay-per-use basis. Resource provisioning for the end user in Cloud computing is done by elasticity of IaaS Cloud as elasticity increases the effectiveness of the Cloud. D Chui et al [5] illustrated that resource provisioning performance is evaluated by the satisfaction factor of Cloud user. This satisfaction can also be affected by the turn around time.

Cloud Computing provides various advantages for workflow deployment as compared to any other computing environment. Some of the advantages are as follows:

- During execution time, Cloud resources for workflow can be changed dynamically. 
- Service requests decide the allocation of resources to a specific workflow.

- Efficient scheduling algorithms assign optimum computing resources at the beginning of workflow execution.

The components used in the deployment of the workflow environment are depicted in Figure 1.

Figure 1 shows a Workflow executes by combining the relevant computational resources required for the execution of a single task. The output given by one process is assigned as input for another process and so on. Some examples of scientific workflows are Cyber shake [3], which classifies the threat of earthquakes and is popularly used by California State in US.LIGO [4], is another workflow, used by Epigenomic for analyzing gravitational waves.

Several large scale scientific workflow algorithms have been proposed for the IaaS Cloud Computing platform. Study of these algorithms reveals that resource provisioning methods have to still develop the best allocation technique for workflow scheduling.

P Vinothiya lakshmi et al [6] proposed the QoS clustering method with the Auction technique using the E-MCA technique. They proposed two techniques i) Measuring QoS for resource allocation ii) Double sided enhanced combinative auction technique for efficient dynamic resource provisioning. The results show that these techniques are attaining minimum execution time and cost with multiple QoS parameters.

In this paper, the authors have surveyed various state-of-the-art scheduling techniques for workflow resource provisioning system and proposed a model for efficient dynamic resource provisioning considering factors like elasticity, resource-abundance, and QoS in terms of cost and execution time.

The paper is distributed into various sections. Section 1 contains the introduction followed by section 2 which provides the background by discussing the related works. Then section 3 outlines the proposed work along with the problem definition and then evaluation of the proposed work with the help of experimental results. Finally, section 4 concludes the papers and provides the future scope of this research work.

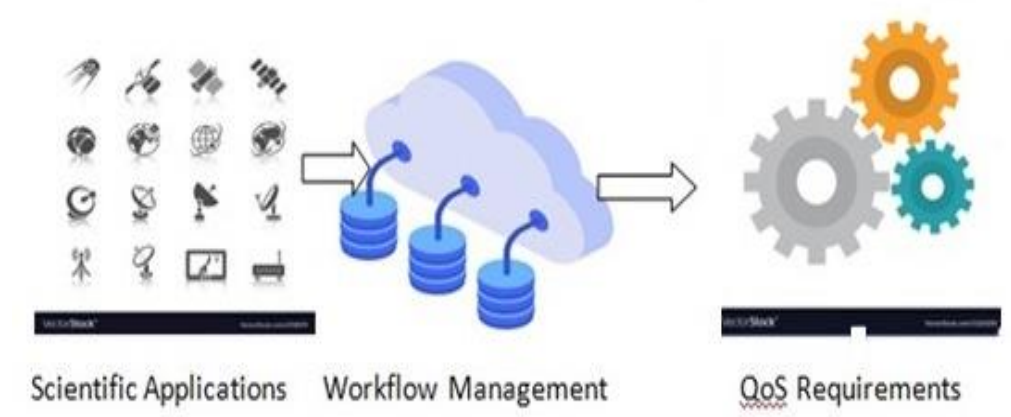

Figure 1: A High-Level View of a Cloud Workflow Execution Environment.

\section{BACKGROUND}

NIST [1] defines Cloud as an on-demand network access for providing computing resources like network servers, storage, and application to multiple users simultaneously. As defined by NIST, Cloud Computing architecture is formed by three layers (IaaS, PaaS, and SaaS). IaaS Cloud layer is the bottom layer of the Cloud framework and it is required to deploy the workflows.

Workflow can be defined as a business process modelling tool. A scientific workflow is used to solve difficult 
scientific tasks. In these workflows, various scientific theories are proved by simulating the experiments. A Scientific workflow solves $\mathrm{n}$ tasks where $1^{\text {st }}$ task generates three outputs, let's say $\mathrm{x}, \mathrm{y}, \mathrm{z}$. Tasks $\mathrm{x}, \mathrm{y}, \mathrm{z}$ cannot process until the $1^{\text {st }}$ task has completed its execution and so on.

Figure 2 shows scientific workflow distributes each complex computational problem into various sub-problems and solves them independently. Montage workflow [8] has introduced an astronomy application that generates manual mosaics of the sky from the set of input images. Astronomers generate a mixed image of a sky region which can be measured by different wavelengths. Workflow scheduling is considered as NP-hard problem; so, optimum results cannot be generated[13].

Several workflow algorithms aim to perform under minimum execution time but the cost of IaaS utilized resources is always neglected. Buyya et al [10] proposed a PSO technique to maintain the cost of workflow under a definite deadline. Kaur et al [9] proposed a hybrid genetic algorithm that uses the PEFT scheduler to minimize the cost to maintain the execution time under the given deadline. Maria Carla et al [10] proposed a scheduling framework that handles the performance of the Infrastructure of the Cloud.

Ananthi et al [20] proposed a method for reducing the estimated cost and increasing the execution time of the workflow. They have proposed a Jobra engine and extended the controller algorithm. Wechen et al [11] have proposed a meta-heuristic technique that has been applied on parallel application working in a scientific workflow to schedule a group of tasks in a dynamic environment [11].

Abrishami et al [21] proposed a partial critical path method for workflows working on a single workflow instance in IaaS. Based on the heuristic scheduling they tried to minimize the execution cost.

After surveying various state-of-art algorithms for workflow it has been observed that the researchers have always neglected the factor of elasticity in Cloud and considered a static set of VM for execution which can be smoothly applied to Grid or Clusters.

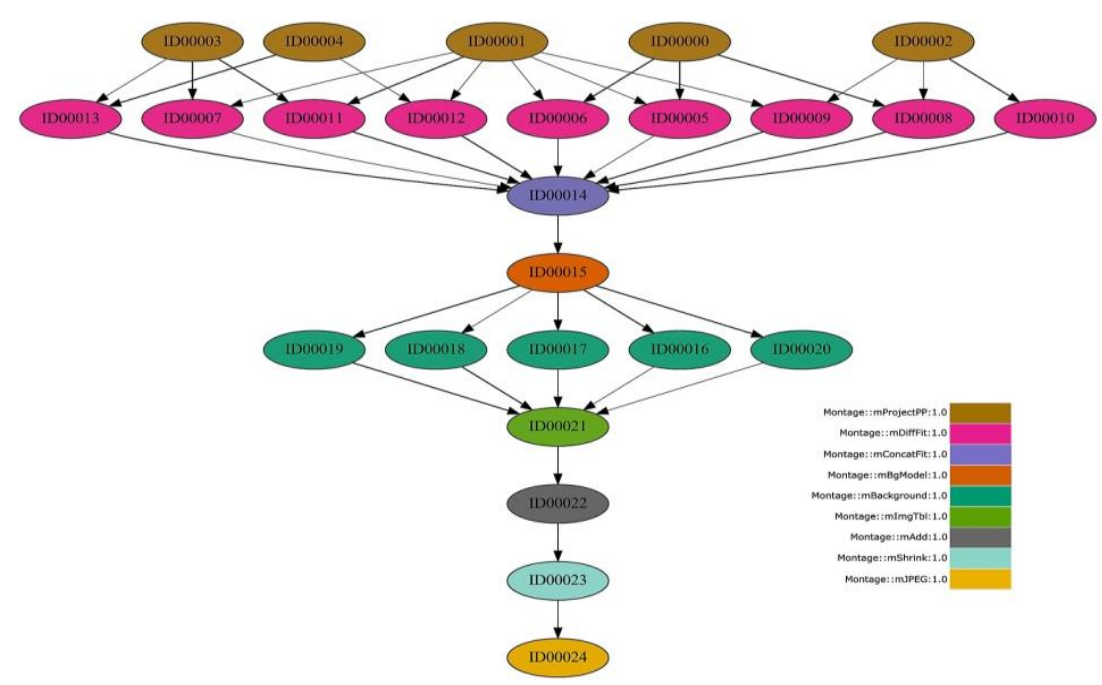

Figure 2: Sample Montage V2 Workflow [7].

\section{PROPOSED WORK}

This research work proposes a scheduling method that works on meta- heuristic technique so that within a deadline it should reduce the workflow execution time. The workflow uses the Cloud Computing environment and performs the Resource 
allocation strategy and VM mapping. In earlier research in this field, workflows were deployed on Grid Computing environment and cluster computing environment, which was applicable in the scheduling phase. Cluster and Grid contain a static group of computing resources which makes it easier to schedule these workflows [12].

Cloud Computing paradigm as a group of VMs, challenges the platform for workflow scheduling. Scheduling in VMs varies in terms of execution time. So, workflow, when deployed over Cloud environment may affect the performance and also the application may miss the deadline.

The authors in this paper have proposed a method called PSO-HOM (particle swarm optimization for homogenous environment) that is based on the fundamentals of PSO (particle swarm optimization). It works like a cluster of particles moving through a medium and interacting with each other to find the best search path. This method works better in terms of execution time than other related algorithms. This method also times itself in the same parameters.

In this proposed Work, both scheduling and resource allocation are combined and defined as an optimum solution for workflow management. PSO solves these problems and maps the resources. After mapping, the algorithm should give higher accuracy meeting the deadline at a low cost.

\subsection{Problem Definition}

Scheduling algorithms and resource allocation methods may have different purpose. The proposed work is applicable to scheduling a workflow on the Infrastructure layer to minimize the total execution cost before the deadline. A scheduler $\mathrm{S}$ $=(\mathrm{V}, \mathrm{M}, \mathrm{TEC}, \mathrm{TET})$ is a set of computing resources where TEC (eq.1) is total execution cost and TET (eq.2) is total execution time. $V=V_{1}, V 2, V_{3} \ldots . . V_{n}$ is a set of $V M$ 's that are used during resource allocation. Each $V_{i}$ is shown as $V_{v i}$ with an estimated start time $\mathrm{ST}_{\mathrm{vi}}$ and end time $\mathrm{ET}_{\mathrm{vi}}$.

Task $\mathrm{t}_{\mathrm{i}}$ scheduled on resource $\mathrm{r}_{\mathrm{j}}$ within starting time $\mathrm{ST}_{\mathrm{ir}}$ and end time $\mathrm{ET}_{\mathrm{ti}}$

$$
\begin{aligned}
& \left.\mathrm{TEC}=\sum_{1=1}^{R} \operatorname{CvMnX} \text { XLETri }-\frac{\text { LSTri }}{\mathrm{E}}\right](1) \\
& \text { TET }=\text { MAXIMUM }\left\{\mathrm{ET}_{\mathrm{ti}}: \mathrm{t}_{\mathrm{i}} \mathrm{ET}\right\}
\end{aligned}
$$

Scheduler must work to provide minimum TEC and TET should not cross the workflow deadline.

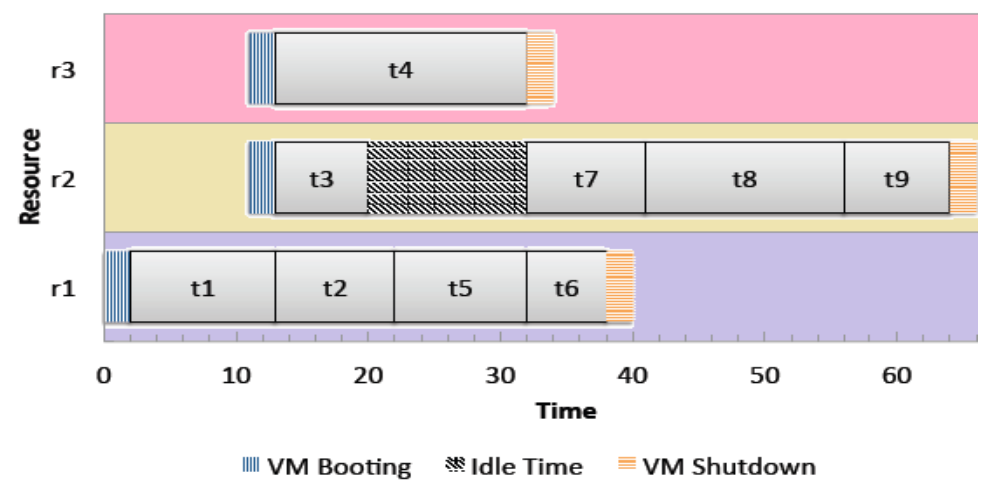

Figure 3: Schedule Generated for the Workflow.

\subsection{Proposed Approach}

In this work, firstly PSO method is used for defining the goodness of a computing resource (particles). It uses a fitness 
function for the evaluation. But PSO does not work well for optimization problem within constrained value due to the heterogeneous features of the VMs. In this work, the authors have proposed a PSO-HOM method to handle constrained optimization problems, which considers the homogenous environment in which single type of VMs should be considered. Firstly, two solutions for workflow check for their fitness by using the fitness function. The one with better fitness will be selected for workflow scheduling. Secondly, if any other solution is feasible then that will be selected. Thirdly, if both are infeasible then the one with a smaller constraint value will be selected.

\section{Algorithm-Scheduler Generator}

Step 1: define SCHEDULER_GENERATOR (T,R initial $_{\text {, }}$ pos[T])

Step 2: V=null

Step 3: $M=$ null

Step 4: TEC $=0$

Step 5: TET $=0$

Step 6: Compute Exe.Time [T]X $\mathrm{R}_{\text {initial }}$

Step 7: Compute Transfer time [T] X[T]

Step 8: while $\mathrm{j}=0$ to [Total time $]-1$

Step 9: $t_{j}=T[j]$

Step 10: $r_{\text {pos }}[j]=R_{\text {initial }}(\operatorname{pos}[j])$

Step 11: if $t_{i}$ has no parents

Step 12: $\mathrm{ST}_{\mathrm{ti}}=\mathrm{ST}_{\mathrm{rpos}[\mathrm{i}]}$

Step 13: else

Step 14: $\mathrm{ST}_{\mathrm{ti}}=$ MAXIMUM $\left.\left(\mathrm{ET}_{\mathrm{tp}}: \mathrm{t}_{\mathrm{p}} £ \operatorname{parent}\left(\mathrm{t}_{\mathrm{i}}\right)\right), \mathrm{ST}_{\mathrm{rpos}[\mathrm{i}]}\right)$

Step 15: end if

Step 16: exe (Execution Time[j] pos[j])

Step 17: for each child $t_{c}$ of $t_{i}$ parents do

Step 18: if $t_{c}$ (child is mapped to resource $r_{j}$

Step 19: transfer resource $=$ transfer time[j]

Step 20: end for

Step 21: $\mathrm{PT}_{\mathrm{ti}} \operatorname{pos}[\mathrm{j}]=$ execution time+ transfer

Step 22: $\mathrm{ET}_{\mathrm{ti}}=\mathrm{PT}_{\mathrm{ti}}+\mathrm{ST}_{\mathrm{ti}}$

Step 23: $\mathrm{mt}_{\mathrm{i}}=\left(\mathrm{t}_{\mathrm{i}}, \mathrm{rpos}[\mathrm{i}], \mathrm{ST}_{\mathrm{ti}}, \mathrm{ET}_{\mathrm{ti}}\right)$

Step 24: $M=M U ~ M t_{i}$ 
Step 25: if $\operatorname{rpos}[i] £ R$ then

Step 26: $\mathrm{ST}_{\mathrm{r}}=\mathrm{MAXIMUM}\left(\mathrm{ST}_{\mathrm{t}}\right.$, boottime $)$

Step 27: R=RU $\{\operatorname{rpos}[i]\}$

Step 28: end for

Step 29: calculate TEC ( $\left.\mathrm{eq}^{\mathrm{n}} 1\right)$

Step 30: calculate TET ( $\left.\mathrm{eq}^{\mathrm{n}} 2\right)$

Step 31: $S=(V, M, T E C$, TET $)$

Step 32: end for

\subsection{Result Analysis}

The performance of the proposed method has been evaluated using workflow simulation tool CloudSim[14]. Two workflows of different fields have been considered in this paper: Montage and Cybershake. These structures have different computational features. Montage requires less CPU processing on the other hand Cybershake consumes more memory to process [15].

Here the workflow environment uses two algorithms. IaaS Cloud Partial Critical Path (IC-PCP)[16] and SCS[17] algorithms have been considered as base algorithms to evaluate the proposed work. IC-PCP helps to schedule a single type of workflow for minimizing the execution cost. While SCP[17] works dynamically to schedule the set of workflows. It groups similar tasks and considers it as one. Yu et al[18] have proposed a deadline constraint which has been used as a parameter in the workflow environment. Two different deadline constraints have been used in this work. Deadline is evaluated and the values lie between slower and faster runtime. Here the selection is done by the scheduler and it first schedules the workflow with slower execution time and then schedules the minimum cost VM from the Cloud. Secondly, it selects the workflow with fastest execution time for scheduling the minimum cost VM.

The scheduler used for VM selection (particles) with $\mathrm{n}$ tasks is having different parameters. The result calculation for average workflow execution is done 10 times for each value.

\subsection{Performance Evaluation}

The dotted line on each panel in Figure 4 is showing the deadline value in a given interval of time. For montage workflow, IC-PCP fails to achieve the deadline value in Q1 (first quadrant) by IC-PCP is very high than the deadline whereas Cyber shake IC-PCP shows the best performance at deadline value. PSO generates the fastest schedule in a deadline. In both cases, PSO-HOMworkflow is making a large variation. 


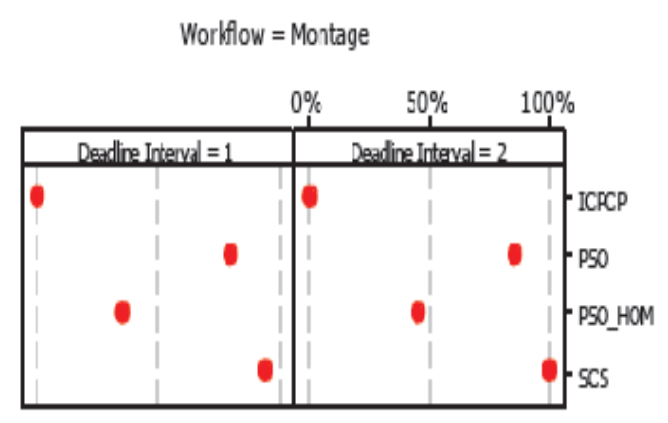

Worlflow $=$ CyberShake

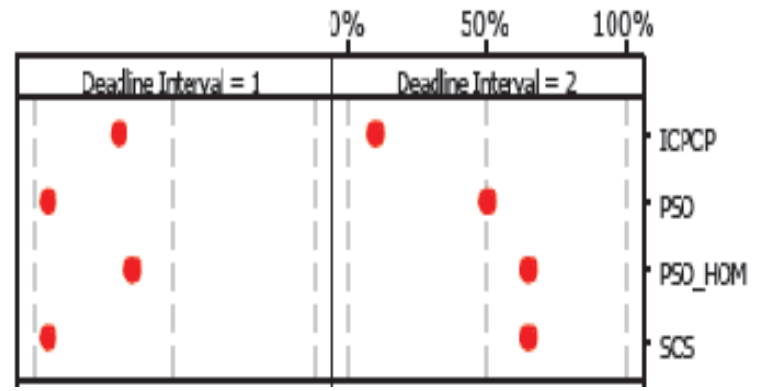

Figure 4: Deadline Meeting of Each Workflow with Their Interval.

\subsection{Cost Evaluation}

For our two well-known workflows we have shown our average execution cost in figure 3.2. Mean has been shown and the algorithm should be able to show the cost-efficient scheduling neglecting the expense of long execution time. We have shown cost comparison among those methods which meet a particular deadline constraint.

In Montage workflow IC-PCP execution cost shows lower value in all 4 deadline intervals, PSO generates the lowest cost on deadline interval 1. Our method PSO-HOM is showing the lowest cost in deadline interval 2. From the results, it is clear that PSO based methods are performing better as compare with SCS and IC-PCP. Cost generated by all the algorithms is different from each other while comparing expensive and cheapest one.
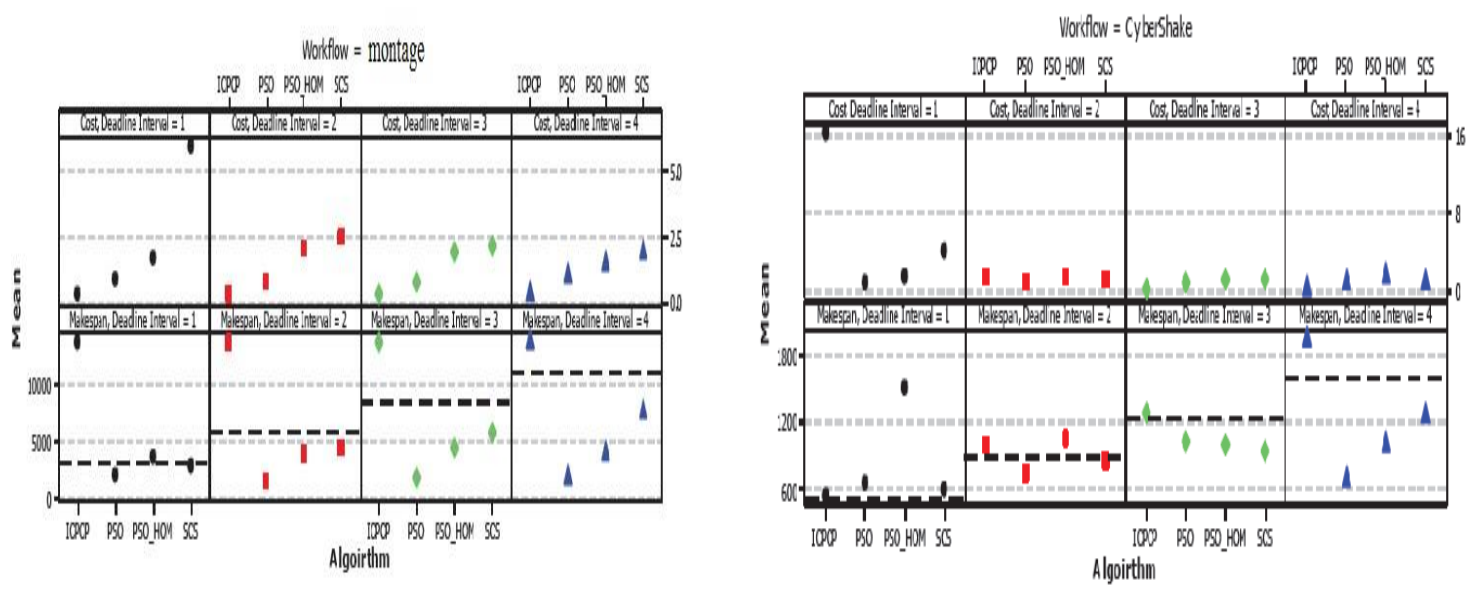

Figure 5: Mean (Ms) and Mean Cost (USD) For Each Workflow under A Deadline Constraint.

\section{CONCLUSIONS AND SCOPE OF FUTURE WORKS}

This paper depicts the execution of resource provisioning algorithm on scientific workflow at IaaS layer on Cloud. The objective was to reduce the execution cost under a deadline constraint by the user that has been solved by implementing a PSO based algorithm. The proposed work associates with IaaS Cloud characteristics like pay-as-per-use and elasticity of resources in a dynamic manner. This paper also proposes the performance evaluation under VM provisioning delays. The simulation has been conducted of two well-known workflows (Montage and Cyber shake) and the proposed method was performed well under the existing algorithms. As in this work, the authors have tested the proposed method with IC-PCP and SCP, so, the future work will be the scalability of workflow in terms of the number of tasks as large scale VM provisioning delays may not perform well when deployed under the real Cloud Computing environment. 


\section{REFERENCES}

1. P. Mell and T. Grance, "The NIST definition of cloud computing," 2011.

2. Y. Gil, E. Deelman, M. Ellisman, T. Fahringer, G. Fox, C. Goble, M. Livny, L. Moreau, and J. Myers, "Examining the Challenges of Scientific Workflows, "IeeeComput., vol. 40, no. 12, pp. 26-34,2007.

3. R. Graves, T. H. Jordan, S. Callaghan, E. Deelman, E. Field, G. Juve, C. Kesselman, P. Maechling, G. Mehta, K. Milner et al., “Cybershake: A physics-based seismic hazard model for southern california,” Pure and Applied Geophysics, vol. 168, no. 3-4, pp. 367-381, 2011.

4. Jakkula, S. U. B. O. D. H., and G. SRINIVASA Sharma. "Analysis of a cross flow heat exchanger using optimization techniques." (2007).International Journal of Applied Engineering Research and Development (IJAERD) 4. 6, Dec 2014, 110.

5. A. Abramovici, W. E. Althouse, R. W. Drever, Y. Gursel," S. Kawamura, F. J. Raab,D. $\quad$ Shoemaker, L. Sievers, R. E. Spero, K. S. Thorne et al.,Ligo: The laser inter-ferometer gravitational-wave observatory, 'Science, vol. 256, no. 5055, pp. 325-333, 1992.

6. D. Chiu, Elasticity in the cloud, ACM Crossroads 16 (3) (2010) 3-4.

7. $\quad P$. Vinothiyalakshmi and $R$. Anitha, "Efficient dynamic resource provisioning using auction based technique with improved QoS in cloud environment, " 2017 International Conference on Computational Intelligence in Data Science(ICCIDS), Chennai, 2017, pp. 16.

8. GIRGIS, MOHEB R., et al. "HETEROGENEOUS MULTIPROCESSOR TASK SCHEDULING USING GENETIC AND SIMULATED ANNEALING ALGORITHMS AND THEIR HYBRIDIZATION."International Journal of Computer Science Engineering and Information Technology Research (IJCSEITR) 4. 6, Dec 2014, 5162.

9. Cotes-Ruiz, Iván\& Prado, Rocío\& Galan, Sebastian \& Muñoz- Expósito, José \& Ruiz Reyes, Nicolas. (2017). Dynamic Voltage Frequency Scaling Simulator for Real Workflows Energy-Aware Management in Green Cloud Computing. PLoS ONE.

10. “LIGO Detection Portal,” https://www.ligo.caltech.edu/detection, accessed: December 2019.

11. G. Kaur and M. Kalra, "Deadline constrained scheduling of scientific workflows on cloud using hybrid genetic algorithm, "2017 $7^{\text {th }}$ International Conference on Cloud Computing, Data Science \& Engineering - Confluence, Noida, 2017, pp. 276280.

12. Rai, S. O. N. A. L., S. K. Mishra, and M. U. K. E. S. H. Dubey. "Teacher learning based optimization of assignment model." Int. J. Mech. Prod. Eng. Res. Dev 3.5 (2013): 6172.

13. Maria A. Rodriguez and RajkumarBuyya. "A Taxonomy and Survey onScheduling Algorithms for Scientific Workflows in IaaS Cloud Computing Environments." Concurrency and Computation: Practice and Experience, DOI:10.1002/cpe.4041, 2016.

14. Wechen,Talbi, Metaheuristics: from design to implementation.John Wiley \& Sons, 2009, vol. 74.

15. H.-H. Li, Y.-W. Fu, Z.-H.Zhan, and J.-J. Li, "Renumber strategyenhanced particle swarm optimization for cloud computing resourcescheduling, " in Proceedings of the IEEE Congress on EvolutionaryComputation (CEC). IEEE, 2015, pp. 870-876.

16. PADMAVATHI, M., and SHAIK MAHABOOB BASHA. "A CONCEPTUAL ANALYSIS ON CLOUD COMPUTING ACO LOAD BALANCING TECHNIQUES. "International Journal of Computer Science and Engineering (IJCSE) 6. 4, Jun - Jul 2017; 39-48

17. K. Deb, A. Pratap, S. Agarwal, and T. Meyarivan, "A fast and elitist multiobjective genetic algorithm: Nsga-ii," IEEE Transactionson Evolutionary Computation, vol. 6, no. 2, pp. 182-197, 2002.

18. W. Chen and E. Deelman, "WorkflowSim: A toolkit for simulatingscientific workflows in distributed environments, " 2012 IEEE $8^{\text {th }}$ International Conference on E-Science, Chicago, IL, 2012, pp. 1-8.

19. M. Malawski, K. Figiela, M. Bubak, E. Deelman, and J. Nabrzyski, "Scheduling multilevel deadline-constrained scientific 
workflows onclouds based on cost opti-mization, " Scientific Programming, vol.2015.

20. S. Abrishami, M. Naghibzadeh, and D. H. Epema, "Deadline-constrained work-flow scheduling algorithms for Infrastructure as aService Clouds, " Future Genera-tion Computer Systems, vol. 29, no. 1,pp. 158-169, 2013.

21. "A performance study on the VM startup time in the cloud," in Proceedings of the Fifth IEEE International Conference on CloudComputing (CLOUD). IEEE, 2012, pp. 423-430.

22. J. Yu, R. Buyya, and K. Ramamohanarao, "Workflow schedulingalgorithms for grid computing," in Metaheuristics for Scheduling in Distributed Computing Environ-ments. Springer, 2008, pp. 173-214.

23. M. Malawski, G. Juve, E. Deelman, and J. Nabrzyski, "Cost-anddeadline-constrained provisioning for scientific workflow ensemblesin IaaS clouds," in Pro-ceedings of the International Conference onHigh Performance Computing, Networking, Storage and Analysis (SC). EEE, 2012, p. 22.

24. Ananthiet,Z. Zhu, G. Zhang, M. Li, and X. Liu, “Evolutionary multi-objective workflow scheduling in cloud, ”IEEE Transactions on Parallel and Distributed Systems, vol. 27, no. 5, pp. 1344-1357, 2016.

25. S. Abrishami, M. Naghibzadeh, and D. H. Epema, "Deadline-constrained work-flow scheduling algorithms for Infrastructure as aService Clouds, " Future Generation Computer Systems, vol. 29, no. 1, pp. 158-169, 2013. 

